\title{
DIFICULTADES EN EL AUTOCONCEPTO DESDE LA NIÑEZ A LA JUVENTUD EN PERSONAS CON BAJA VISIÓN
}

\author{
M. Dolores López-Justicia \\ Departamento de Psicología Evolutiva y de la Educación \\ Universidad de Granada (España) \\ E-mail para correspondencia: dlopezj@ugr.es \\ https://doi.org/10.17060/ijodaep.2017.n1.v3.999
}

Fecha de Recepción: 13 Febrero 2017

Fecha de Admisión: 1 Abril 2017

\section{RESUMEN}

El presente estudio se planteó como objetivo principal determinar si niños/as, adolescentes y jóvenes afectados de baja visión, mostraban diferencias en el autoconcepto respecto a iguales sin dificultades visuales. La población total de participantes se dividió en cuatro grupos de edades: de 4 a 7 años, de 8 a 11, de 12 a 17 y de 18 a 30 años. Los resultados de los distintos análisis efectuados pusieron de manifiesto que en los cuatro grupos de edad se obtuvieron puntuaciones más bajas en algunas dimensiones del autoconcepto en los afectados por baja visión, comparados con sus iguales de visión normal. Esto lleva a poner de relieve la conveniencia de actuar en el ámbito psico-educativo para incidir en el desarrollo adecuado del autoconcepto desde los primeros años escolares. Se concluye haciendo una llamada de atención a los docentes que intervienen en su educación con el fin de proporcionar apoyo en las áreas curriculares que lo requieran, e introducir cambios metodológicos en el proceso de enseñanza-aprendizaje que permita ayudar a estos niños/as y jóvenes a mejorar su autoconcepto y rendimiento escolar, así como facilitar su inclusión social.

Palabras clave: autoconcepto; baja visión; niñez; juventud; educación.

\section{ABSTRACT}

Self-concept difficulties from childhood to youth in people with low vision

The present study had as its main objective to determine if children, adolescents, and young people with low vision, showed differences in the self-concept with respect to equal without visual difficulties. The total population of participants was divided into four age groups: 4 to 7 years, 8 to 11,12 to 17 and 18 to 30 years. The results of the various analyzes carried out showed that those affected by low vision obtained lower scores in some dimensions of the self-concept in the four age groups, compared with peers of normal vision. This leads to highlight the need to act in the psycho- 


\section{DIFICULTADES EN EL AUTOCONCEPTO DESDE LA NIÑEZ A LA JUVENTUD EN PERSONAS CON BAJA VISIÓN}

educational field to influence the adequate development of the self-concept from the early school years. It is concluded by making a call of attention to teachers involved in their education, in order to provide support in the curricular areas that require it and introduce methodological changes in the teaching-learning process that will help these children and young people to improve their selfconcept and academic performance, as well as to facilitate their social inclusion.

Keywords: self-concept; low vision; childhood; youth; education.

\section{INTRODUCCIÓN}

La preocupación por el autoconcepto en el contexto educativo se remonta a los años 50 del siglo $X X$, cuando los profesionales de la educación empezaron a tomar conciencia de su importancia en el progreso académico. Es por ello, que la psicología educativa ha estudiado la relación entre el autoconcepto y el rendimiento escolar tanto en niños (González- Pienda et al., 2003), como adolescentes y universitarios (Torres et al., 2005; Gargallo, Garfella, Sánchez, Ross, y Serra, 2009), encontrándose que contribuye a la motivación e incide directa y significativamente en los logros académicos.

El autoconcepto ha sido descrito como una organización cognitivo afectiva que influye en la conducta, actuando como filtro o mediador de la información que se recibe del medio (González y Touron, 1994), relacionándose con el bienestar personal y psicosocial, al contribuir a la salud y equilibrio mental (Fuentes, García, Gracia, y Lila, 2011; Goñi, Esnaola, Rodríguez, y Camino, 2015). De ahí que puntuaciones más altas en este constructo permiten enfrentarse en mejores condiciones a las consecuencias de la discapacidad, mientras que puntuaciones más bajas repercuten negativamente en la salud física y se relacionan con depresión e infravaloración personal (Smith, NolenHoeksema, Fredrickson, y Loftus, 2003).

El autoconcepto es entendido como un conjunto organizado de actitudes que el individuo tiene hacia sí mismo (Harter, 1999). Encierra tres elementos fundamentales: a) identidad del sujeto 0 autoconcepto (autoimagen), referido a la percepción que tiene la persona de sí misma en relación a los aspectos cognitivos; b) autoestima, que tiene que ver con el valor que el individuo atribuye a su particular manera de percibirse (González y Tourón, 1994), estando ésta cargada de connotaciones evaluadoras, afectivas y emotivas; c) componente comportamental, en cuanto que el autoconcepto influye en la conducta y la condiciona. Autoconcepto y autoestima son conceptos que se implican y complementan, por lo que un autoconcepto positivo llevaría a una autoestima positiva y viceversa. Ambos términos se pueden utilizar indistintamente puesto que uno y otro incluyen elementos cognitivos, afectivos y comportamentales (Machargo, 1997).

En un comienzo se entendía el autoconcepto como un constructo simple y estático, sin embargo, en la actualidad los modelos con mayor aceptación lo conciben como un constructo multidimensional y jerárquico (González-Pienda et al. 2003).

Conviene resaltar que el autoconcepto, se desarrolla con los años de acuerdo a la experiencia acumulada (García y Musitu, 2001) y, aunque relativamente estable, está sometido a cambios en los aspectos más específicos como resultado de las distintas experiencias vividas. De este modo la característica más destacada del autoconcepto infantil de los niños de 5 a 11 años de edad es que éste se encuentra en pleno proceso de desarrollo, ya que en estos años se va implantando el yo y el sentimiento de sí mismo, por ello, cualquier alteración que se produzca a este nivel puede repercutir negativamente en su futuro autoconcepto. Durante esta etapa el niño necesita experimentar cierta autonomía, diferenciarse de los demás afianzando su individualidad, pero a la vez necesita tener confianza en su familia y en su medio para refugiarse y encontrar apoyo. De acuerdo con Machargo (1991), el autoconcepto se construye a merced de las relaciones con las personas signi- 
ficativas del entorno (familia y escuela) y como consecuencia de las experiencias positivas o negativas que se hayan experimentado.

En personas con discapacidad el yo crece y se desarrolla igual que en el resto, pero en muchos casos esta población queda expuesta desde la infancia a rechazo social, prejuicio o actitudes negativas que pueden desvalorizar la imagen de sí mismos (Polo y López-Justicia, 2012); a lo que se suman las dificultades individuales del proceso de adaptación a la discapacidad. En esta línea, Pérez y Garaigordobil (2007a, 2007b), señalan que el desarrollo del autoconcepto es importante en estas personas, pues la comparación social desempeña un papel fundamental en la autoestima. En este sentido si se genera información negativa derivada de este proceso de comparación, sobre la base la percepción de limitaciones significativas en el funcionamiento de algunas de las habilidades adaptativas, ello supondría una amenaza para la autoestima y en consecuencia, para el autoconcepto.

En el caso de niños que padecen una disminución visual se ha sugerido que muchos padres 0 familiares cercanos suelen experimentar ciertas conductas típicas cuando nace un niño con esta problemática, o lo perciben incompleto o imperfecto (Beaty, 1992); otros desarrollan conductas de sobreprotección o alta permisividad hacía sus hijos que impide a éstos tener una referencia clara de lo que deben y no deben hacer, o de lo que realmente les piden. Dado que existen pocas investigaciones que aborden el campo del autoncepto en niños y jóvenes afectados de baja visión la cuestión fundamental que se planteó este estudio era determinar si niños/as de 4 a 11 años de edad, adolescentes de 12 a 17 años y jóvenes de 18 a 30, que padecían baja visión, mostraban dificultades en su autoconcepto frente a otros sin problemas visuales. Esta evaluación permitirá adoptar medidas psico-educativas que ayuden a los profesionales a actuar para mejorar la autopercepción de esta población, facilitando de esta manera su ajuste personal y social.

La definición de baja visión que se utiliza es la facilitada por la OMS (2008) en la que aludía a aquellos que presentan una deficiencia en el funcionamiento visual que aún después de tratamiento y/o corrección óptica mantienen una agudeza visual (AV) comprendida entre 6/18 y percepción de luz, 0 un campo visual (CV) menor de 20 grados, pero que usan la visión, o son capaces potencialmente de hacerlo, para la planificación o ejecución de una tarea.

\section{ESTUDIO 1}

El grupo 1 estaba constituido por 30 niños y niñas de 4 a 7 años: 15 con dificultades visuales (7 niños y 8 niñas) y 15 sin ellas. Los primeros tenían una A.V. que oscilaba entre 1/4 y 1/20.

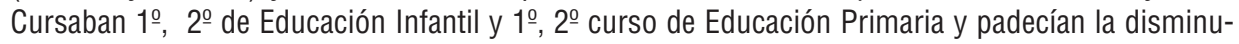
ción visual desde el nacimiento, presentando patologías diversas. Los niños/as sin dificultad visual estaban distribuidos de forma idéntica en cuanto a número, edad y sexo.

\section{Materiales}

Para evaluar el autoconcepto se utilizó la escala PAI (Percepción del Autoconcepto Infantil), por su fácil aplicación para niños de 4 a 7 años independientemente de que presenten o no dificultades de visión. Consta de 34 ítems, expresados en escenas en blanco y negro con buen contraste y claramente reconocibles, que representan imágenes familiares alusivas a situaciones de autoconcepto positivo o negativo, entre las que tiene que elegir (Villa y Auzmendi, 1992). La fiabilidad y validez de la escala es elevada: 0.90 (Villa y Auzmendi, 1992) y evalúa diez componentes: a) autonomía (sentimiento de independencia); b) seguridad (confianza en uno mismo en la realización de tareas); c) deportes (valía en la competición); d) familia (cómo se siente en ella); e) aula (cómo se siente en el aula); f) social (relaciones sociales); g) afectividad (cómo se siente por regla general); h) autovalía (en el sentido de competencia individual); i) aspecto físico y j) sentimiento de posesión, de amigos, objetos, etc. 


\section{DIFICULTADES EN EL AUTOCONCEPTO DESDE LA NIÑEZ A LA JUVENTUD EN PERSONAS CON BAJA VISIÓN}

\section{Procedimiento}

Tanto a los niños de baja visión como a los que no padecían dificultades visuales se les aplicó la escala de autoconcepto de forma individual. Para los primeros se utilizó una copia ampliada de la escala de acuerdo al tamaño que eran capaces de ver con comodidad. A los niños con visión normal se les facilitó igualmente una copia (a tamaño normal) impresa de la escala.

\section{Resultados}

Para el análisis de los datos se utilizó el paquete estadístico SPSS 20.0 aplicando la prueba $t$ de Student de diferencia de medias para grupos independientes, más adecuada en función de la homogeneidad de las varianzas.

Comparadas las medias de las puntuaciones obtenidas por los niños de baja visión en cada uno de los 10 aspectos que evalúa la escala PAI, con las medias de las puntuaciones obtenidas por los niños de visión normal los resultados obtenidos se pueden observar en la Tabla 1.

Tabla 1.

Diferencia de medias en autoconcepto entre los niños/as de 4 a 7 años de baja visión (BV) y los de visión normal (VN)

\begin{tabular}{|c|c|c|c|c|c|c|}
\hline \multirow[b]{2}{*}{ Variables } & \multicolumn{2}{|c|}{$\begin{array}{l}\text { Visión } \\
\text { normal }\end{array}$} & \multicolumn{2}{|c|}{ Baja visión } & \multicolumn{2}{|c|}{$\begin{array}{c}\text { Diferencias } \\
\text { Medias }\end{array}$} \\
\hline & $\mathbf{M}$ & DT & $\mathrm{M}$ & DT & $\mathrm{t}$ & $\mathrm{p}$ \\
\hline Afectividad & 10.87 & .83 & 9.67 & 1.18 & $\begin{array}{r}3.22 \\
5\end{array}$ & $.003^{*}$ \\
\hline Aula & 17.60 & 2.13 & 17.07 & 1.75 & .749 & .460 \\
\hline Autonomía & 10.93 & 1.49 & 7.60 & 2.67 & $\begin{array}{r}4.22 \\
8\end{array}$ & $.000^{*}$ \\
\hline Autovalía & 18.13 & 1.73 & 17.53 & 2.17 & .839 & .409 \\
\hline Deportes & 7.13 & .99 & 5.60 & 2.33 & $\begin{array}{r}2.43 \\
4\end{array}$ & $.025 *$ \\
\hline Familia & 7.53 & 1.60 & 6.93 & 2.15 & .867 & .394 \\
\hline Físico & 14.73 & 1.58 & 14.00 & 1.65 & $\begin{array}{r}1.24 \\
4\end{array}$ & .224 \\
\hline Posesión & 14.33 & 1.80 & 12.67 & 2.41 & $\begin{array}{r}2.14 \\
6\end{array}$ & $.041 *$ \\
\hline Seguridad & 6.40 & 1.64 & 5.87 & 1.36 & .971 & .340 \\
\hline Social & 11.33 & 1.11 & 9.67 & 1.50 & $\begin{array}{r}3.46 \\
2\end{array}$ & $.002 *$ \\
\hline
\end{tabular}

*Las diferencias son significativas con una $\mathrm{p}<.05$

$* *$ Las diferencias son significativas con una $\mathrm{p}<.01$ 
La prueba muestra que los niños con baja visión obtienen puntuaciones más bajas que sus iguales de visión normal, obteniendo diferencias significativas en cinco dimensiones del autoconcepto: afectividad, autonomía, deportes, posesión y social. Por el contrario no existen diferencias entre los niños de ambos grupos en percepción en el aula, autovalía, relaciones en la familia, físico y seguridad.

\section{ESTUDIO 2}

El grupo lo integraban 34 niños/as de 7 a 11 años: 17 sin problemas visuales y 17 con baja visión congénita (9 niños y 8 niñas) que cursaban desde $3^{0}$ hasta $6^{0}$ de Educación Primaria. Presentaban distintas patologías y su A.V. oscilaba entre $1 / 4$ y 1/20. Los niños/as sin dificultad visual estaban distribuidos de forma idéntica en cuanto a número, edad y sexo.

\section{Materiales}

Para medir el autoconcepto se utilizó de forma individual la Escala SDQ (Self Description Questionnarie) de Marsh (1984), adaptada por Elexpuru y col. (1992) para niños de 3ㅜㄴ $4^{\circ}$ y $5^{0}$ de Primaria. La fiabilidad y validez es de .74. Mide siete factores específicos: a) habilidad física; b) apariencia física; c) relaciones con los compañeros; d) relaciones con los padres; e) lectura.; f) matemáticas; y g) asignaturas escolares en general.

\section{Procedimiento}

La prueba se aplicó individualmente, facilitando una copia impresa ampliada de la escala, en el caso de los niños/as con baja visión, de acuerdo al tamaño que eran capaces de ver con facilidad.

\section{Resultados}

Se compararon igualmente las medias de las puntuaciones obtenidas por los niños de ambos grupos aplicando la prueba $t$ de Student (Tabla 2).

Tabla 2.

Diferencia de medias en autoconcepto entre los niños/as de 8 a 11 años de baja visión y los de visión normal

\begin{tabular}{|c|c|c|c|c|c|c|}
\hline \multirow[b]{2}{*}{ Variables } & \multicolumn{2}{|c|}{$\begin{array}{l}\text { Visión } \\
\text { normal }\end{array}$} & \multicolumn{2}{|c|}{ Baja visión } & \multicolumn{2}{|c|}{$\begin{array}{c}\text { Diferencias } \\
\text { Medias }\end{array}$} \\
\hline & M & DT & $\mathbf{M}$ & DT & $\mathrm{t}$ & $p$ \\
\hline Habilidad física & 37.41 & 7.13 & 36.70 & 5.82 & .32 & .754 \\
\hline Apariencia física & 34.88 & 7.40 & 36.29 & 7.99 & .53 & .597 \\
\hline $\begin{array}{l}\text { Relación } \\
\text { compañeros }\end{array}$ & 41.88 & 3.84 & 36.53 & 4.87 & 3.56 & $.001 *$ \\
\hline Relación padres & 35.71 & 6.26 & 41.41 & 3.73 & 3.23 & $.003 *$ \\
\hline Lectura & 42.05 & 8.21 & 41.70 & $\begin{array}{r}10.1 \\
8\end{array}$ & .11 & .912 \\
\hline Matemáticas & 38.52 & 6.63 & 38.70 & 8.91 & .07 & .948 \\
\hline Académico total & 40.29 & 5.64 & 39.62 & 6.32 & .32 & .748 \\
\hline $\begin{array}{l}\text { No académico } \\
\text { total }\end{array}$ & 37.47 & 4.51 & 37.73 & 3.81 & .18 & .854 \\
\hline
\end{tabular}




\section{DIFICULTADES EN EL AUTOCONCEPTO DESDE LA NIÑEZ A LA JUVENTUD EN PERSONAS CON BAJA VISIÓN}

Los resultados de este análisis mostraban que los niños/as con baja visión obtienen puntuaciones más bajas que los de visión normal en la dimensión de relación con compañeros, mientras que sus puntuaciones son más altas en la dimensión de relación con padres. No se hallaron diferencias significativas en el resto de dimensiones.

\section{ESTUDIO 3}

El grupo estaba compuesto por 46 adolescentes de entre 12 y 17 años: 23 sin problemas visuales y 17 con baja visión congénita (9 chicos y 8 chicas), matriculados en 2ํㅜ de E.S.0. y Bachillerato. Presentaban patologías diversas y su A.V. oscilaba entre $1 / 4$ y 1/20. Los adolescentes sin dificultad visual estaban distribuidos de forma idéntica en cuanto a número, edad y sexo.

\section{Materiales}

Se utilizó la Escala de Autoconcepto Tennesse (T.S.C.S) de Fits (1965), cuya fiabilidad y validez son adecuadas, .91 (Beaty, 1992; Fitts, 1965). La escala está compuesta por 100 preguntas de las que 45 están expresadas en forma positiva y otras 45 en forma negativa, las 10 preguntas restantes pertenecen a la escala $L$ del MMPI y tienen que ver con la autocrítica. Quienes obtienen altas puntuaciones en la suma total se considera que tienen un nivel de autoconcepto alto; mientras que sucede lo contrario en los que obtienen puntuaciones bajas. Consta de 5 subescalas que evalúan componentes específicos de autoconcepto: personal, familiar, moral, social y físico.

\section{Procedimiento}

En este grupo se daban dos circunstancias entre los participantes: 1. Por una parte se encontraban los jóvenes con baja visión que al ser usuarios de ayudas ópticas (las cuales se reducían a lupas, microscopios y telemicroscopios) podían leer los ítems sin necesidad de usar macrotipos, por lo que se les proporcionó una copia de la escala en su tamaño original; 2. A los jóvenes que no usaban ayudas ópticas se les facilitó una copia ampliada de la escala de acuerdo al tamaño que eran capaces de leer.

\section{Resultados}

Para la comparación de medias en cada una de las dimensiones de autoconcepto, se aplicóo la prueba $t$ de Student (Tabla 3).

Tabla 3.

Diferencia de medias en autoconcepto entre los sujetos de 12 a 17 años de baja visión y los de visión normal

\begin{tabular}{|c|c|c|c|c|c|c|}
\hline \multirow[b]{2}{*}{ Variables } & \multicolumn{2}{|c|}{$\begin{array}{l}\text { Visión } \\
\text { normal }\end{array}$} & \multicolumn{2}{|c|}{ Baja visión } & \multicolumn{2}{|c|}{$\begin{array}{l}\text { Diferen. } \\
\text { Medias }\end{array}$} \\
\hline & $\mathbf{M}$ & DT & $\mathbf{M}$ & DT & $\mathrm{t}$ & $\mathrm{p}$ \\
\hline Autoconcepto Familiar & 26.48 & 5.65 & 26.04 & 4.65 & .278 & .782 \\
\hline Autoconcepto Personal & 23.00 & 4.68 & 23.00 & 3.50 & .000 & 1.00 \\
\hline \multirow[t]{2}{*}{ Autoconcepto Físico } & 23.81 & 2.64 & 21.65 & 2.48 & 2.79 & $.008 *$ \\
\hline & & & & & \multicolumn{2}{|l|}{6} \\
\hline Autoconcepto Social & 21.86 & 4.00 & 20.78 & 3.85 & .907 & .369 \\
\hline Autoconcepto Moral & 24.10 & 3.28 & 23.48 & 3.51 & .600 & .552 \\
\hline
\end{tabular}


Tal como se observa en la Tabla 3, los resultados mostraban que los jóvenes con baja visión obtienen diferencias significativas en el autoconcepto físico puntuando más bajo que los de visión normal. No se establecen diferencias significativas entre los dos grupos en el resto de dimensiones.

\section{ESTUDIO 4}

Participantes 45 personas con edad comprendida entre los 18 y 30 años, distribuidos en dos grupos: 22 afectadas de retinosis pigmentaria y otras patologías (10 hombres y 12 mujeres) y 23 no afectadas. El grupo de afectados por baja visión tenía afectado el campo visual y su A.V. oscilaba entre $1 / 3$ y $1 / 20$.

\section{Procedimiento}

Nuevamente se usó la Escala de Autoconcepto Tennesse (T.S.C.S) de Fits (1965). Los cuestionarios fueron adaptados, en cuanto a contraste y ampliados al tamaño de letra que eran capaces de ver, entre 14 y 16 puntos. En el caso de 5 personas que lo desearon se les enviaron los documentos por correo electrónico y se les remitió a las investigadoras si les surgía cualquier tipo de dudas.

\section{Resultados}

Los datos obtenidos en este trabajo fueron analizados con el programa estadístico S.P.S.S. 20.0, llevando a cabo un análisis de diferencias de medias con la prueba estadística $t$ de Student.

Como puede apreciarse en la Tabla 4 se obtuvieron diferencias significativas en el componente moral-ético, obteniendo puntuaciones más altas los afectados por RP; en el componente familiar, en el que obtuvieron puntuaciones más bajas, al igual que en el componente social; mientras que en los restantes componentes, personal y físico, no se hallaron diferencias significativas.

\section{Tabla 4.}

Diferencia de medias en el auto-concepto de personas con visión normal (VN) y Retinosis pigmentaria (RP)

\begin{tabular}{lcccccc}
\hline Variable & M & DT & M & DT & t & p \\
\hline Autoconcepto & 26.65 & 1.89 & 24.91 & 3.11 & - & $.008^{*}$ \\
Familiar & & & & & 2.278 & \\
Autoconcepto & 22.52 & 3.67 & 23.91 & 2.87 & 1.405 & .411 \\
Personal & & & & & \\
Autoconcepto Físico & 22.65 & 4.13 & 21.41 & 3.54 & - & .971 \\
& & & & & 1.082 & \\
Autoconcepto Social & 22.91 & 4.10 & 22.64 & 2.17 & .281 & $.015^{*}$ \\
Autoconcepto Moral & 21.48 & 3.74 & 23.77 & 2.54 & 2.395 & $.049 *$ \\
Autocrítica & 35.30 & 4.57 & 33.82 & 5.56 & -.981 & .095 \\
\hline *Diferencias significativas p $<.05$ & & & & &
\end{tabular}

\section{DISCUSIÓN Y CONCLUSIONES}

La principal cuestión que se planteó esta investigación era comprobar si niños/as de 4 a 11 años de edad, adolescentes de 12 a 17 y jóvenes de 18 a 30 años afectados de baja visión, tenían difi- 


\section{DIFICULTADES EN EL AUTOCONCEPTO DESDE LA NIÑEZ A LA JUVENTUD EN PERSONAS CON BAJA VISIÓN}

cultades en el autoconcepto respecto a sus iguales. Los datos obtenidos ofrecen un claro soporte a esta cuestión. En primer lugar, los niños con baja visión congénita del primer grupo manifiestan fallos en el autoconcepto en varios aspectos de los evaluados: afectividad, autonomía, deportes, posesión y social. Se puede afirmar, por ello, que son más dependientes de sus padres, se sienten peor que los otros en el aspecto afectivo, esto es, se sienten menos queridos por los demás; muestran un mayor sentimiento de fracaso, o lo que es lo mismo, se perciben como menos válidos en las competiciones 0 en las actividades físico-deportivas; no se sienten bien aceptados por el grupo de iguales, teniendo la percepción de tener menos amigos, incluso sienten poseer menos objetos materiales que los otros. El hecho de que en los restantes aspectos que componen la prueba no se obtengan diferencias, hace pensar que se encuentran bien en el entorno familiar en el que acuden a refugiarse y buscar apoyo ante sus escasos éxitos con los iguales; esto les hace percibirse bien físicamente y sentirse queridos y válidos. Igualmente, en el aula dan muestras de sentirse a gusto, no obstante sus dificultades parecen surgir cuando tienen que competir con los demás.

Los resultados obtenidos por los niños/as del segundo grupo ponían de manifiesto que los niños y niñas con baja visión tenían un autoconcepto más bajo que los otros en cuanto a la relación con sus compañeros, mostrando dificultades para hacer amigos y sentirse queridos por éstos; sin embargo su autoconcepto en relación con los padres era mayor que en los niños sin dificultades visuales. Por lo demás, se observa que se valoran bien físicamente tanto en los aspectos relativos a su apariencia 0 atractivo físico como en lo referido a su habilidad en deportes 0 actividades de este tipo. Igualmente se constata que los niños con baja visión en estas edades no detectan dificultades en su autoconcepto escolar. Tanto en el primero como en el segundo grupo es notable y merece resaltar las buenas relaciones que mantienen con sus familias los niños con baja visión en estos años.

Respecto a los resultados obtenidos por los adolescentes del tercer estudio, llama la atención que los del grupo de baja visión se auto-perciban más negativamente que los del otro grupo en uno de los rasgos que tienen que ver con la discapacidad sensorial-visual: el aspecto físico, por ello se podría sospechar que tal vez han ido recibiendo (a lo largo de sus vidas) una retroalimentación negativa de su deficiencia física y que ésta se ha puesto de relieve en esta etapa de la vida, especialmente conflictiva para los jóvenes. En la adolescencia son notables los cambios físicos y, generalmente, ejercen una gran influencia sobre la configuración de la identidad personal. Resulta llamativo que en las demás dimensiones no aparezcan diferencias, lo que induce a pensar que estos jóvenes se sienten satisfechos con sus valores morales-éticos; tienen una concepción positiva de su personalidad y se sienten integrados en su entorno familiar y social.

En relación al grupo de jóvenes, los datos mostraban que las personas afectadas por RP se valoraban bien y se sentían satisfechas con su conducta moral-ética (Fitts, 1965; 1996). No obstante, sus puntuaciones más bajas en autoconcepto familiar denota cierta insatisfacción con su círculo familiar, dato que pone de relieve su necesidad de que se actúe a este nivel con el fin de cambiar sus percepciones. En cuanto al siguiente componente en el que también obtenían puntuaciones más bajas que las personas sin dificultades visuales, autoconcepto social, se puede deducir una valoración personal no muy adecuada con respecto a los otros.

Los resultados llevan a concluir que los participantes en los 4 estudios (niños/niñas, adolescentes y jóvenes) con baja visión muestran limitaciones en el autoconcepto, frente a personas sin dificultades visuales, considerando pertinente prestar más atención al desarrollo de un autoconcepto adecuado desde los primeros años tras el diagnóstico de la baja visión. Esta propuesta es viable al haberse resaltado que el autoconcepto no es inmutable, sino que los componentes específicos pueden variar a lo largo del tiempo como resultado de la experiencia en el medio social (Coll, 
2002). De los resultados obtenidos se deriva la conveniencia de proponer la adopción de medidas psico-educativas para poner en práctica con este alumnado lo antes posible; medidas a adoptar tanto en los centros educativos como con la familia, dado que la ayuda proporcionada por el grupo de amigos y familiares es fundamental en el estado emocional. De acuerdo con Reinhart (2001), el apoyo y afecto de los amigos se asociaba con mejor adaptación a la pérdida visual y con mayor satisfacción en la vida.

Puesto que se ha vinculado el sentido de competencia personal a puntuaciones más altas en autoconcepto (Goñi et al., 2015) se sugiere potenciar las habilidades personales de cada afectado por baja visión y prestar apoyo en las áreas curriculares en las que muestran más dificultades para que este alumnado pueda avanzar de acuerdo con sus posibilidades. El entrenamiento y la correcta ejecución asociada les llevará a sentirse más competentes, mejorando así su bienestar personal y aceptación social. No se puede perder de vista que casi toda la muestra tiene dificultades en la dimensión social. Conviene, asimismo, utilizar el uso de aquellos recursos ambientales, ópticos y tecnológicos que facilitan el acceso al proceso de enseñanza y aprendizaje, potenciando su autonomía, independencia y movilidad por el aula, el centro y el entorno.

Somos conscientes de que los resultados que ofrece este estudio requieren de investigaciones adicionales, debido a la reducida muestra de éste. Sin embargo, pensamos que puede ser un paso importante en el propósito de mejorar el autoconcepto de estos niños y jóvenes desde los primeros años escolares previniendo posteriores desajustes personales y sociales en los mismos.

\section{REFERENCIAS}

Beaty, L.A. (1992). Adolescent Self-Perception as a Function of Vision Loss. Adolescence, 27(107), 707-714.

Coll, C. (2002). Constructivismo y Educación. En C. Coll, J. Palacios, y A. Marchesi, Desarrollo Psicológico y educación, Vol. 2, Psicología de la educación escolar. Madrid: Alianza Editorial.

Elexpuru, I., Garma, A., Marroquín, M., y Villa, A. (1992): Autoconcepto y Educación. Teoría, medida y práctica pedagógica. Bilbao: Gobierno Vasco.

Fitts, W. H. (1965). Tennessee Self-Concept Scale: Manual. Nashville, Tennessi: Counselor recording and Tests.

Fitts, W.H. y Warren, W.L. (1996). Tennessee Self-Concept Scale: Manual. Western Psychological Services.

Fuentes, M.C., García, J.F., Gracia, E., y Lila, M. (2011). Autoconcepto y ajuste psicosocial en la adolescencia. Psicothema, 23(1), 7-12.

García, F., y Musitu, G. (2001). AF5- Autoconcepto Forma 5 (2a ed.). Madrid: TEA Ediciones.

Gargallo, B., Garfella, P. R., Sánchez, F., Ros, C. y Serra, B. (2009). La influencia del autoconcepto en el rendimiento académico en estudiantes universitarios. Revista Española de Orientación y Psicopedagogía, 20(1), 16-28.

González-Pienda, J.A., Núñez, J.C., Álvarez, L., Roces, C., González-Pumariega, S., González, P., y Bernardo, A. (2003). Adaptabilidad y cohesión familiar, implicación parental en conductas autorregulatorias, autoconcepto del estudiante y rendimiento académico. Psicothema, 15(3), 471477.

Goñi, E., Esnaola, I., Rodríguez, A., y Camino, I. (2015). Personal self-concept and satisfaction with life in adolescence, youth and adulthood. Psicothema, 27(1), 52-58.

González, M.C. y Tourón, J. (1994): Autoconcepto y Rendimiento Escolar. EUNSA, Pamplona.

Harter, S. (1999). The construction of self: A developmental perspectives. New York: The Guilford Press. 


\section{DIFICULTADES EN EL AUTOCONCEPTO DESDE LA NIÑEZ A LA JUVENTUD EN PERSONAS CON BAJA VISIÓN}

Machargo, S.J. (1991). El profesor y el autoconcepto de sus alumnos. Teoría y Práctica. Madrid: Escuela Española.

Machargo, S.J. (1997). Programa de actividades para el desarrollo de la Autoestima. (Vol. 2). Madrid: Escuela Española.

Marsh, H.W. (1984). Relations among dimensions of self-attribution, dimensions of self-concept and academic achievement. Journal of Educational Psychology, 76, 1291-1308.

Organización Mundial de la Salud. (2008). Clasificación Internacional del funcionamiento de la discapacidad visual y de la salud. Ginebra (Suiza).

Pérez, J.I. y Garaigordobil, M. (2007a). Deficiencia auditiva: autoconcepto, autoestima y síntomas psicopatológicos. Análisis y modificación de conducta, 33(148), 159-183.

Pérez, J.I. y Garaigordobil, M. (2007b). Discapacidad motriz: autoconcepto, autoestima y síntomas psicopatológicos. Estudios de Psicología, 28(3), 343-357.

Polo-Sánchez, $M^{\mathrm{a}}$.T., y López-Justicia, Mª.D. (2012). Autoconcepto de estudiantes universitarios con discapacidad visual, auditiva y motora. Revista Latinoamericana de Psicología, 44 (2), 8798.

Reinhardt, J.P. (2001). Effects of positive and negative support received and provided on adaptation to chronic visual impairment. Applied Developmental Science, 5(2), 76-85.

Smith, E., Nolen-Hoeksema, S., Fredrickson, B. y Loftus, G. (2003). Introducción a la Psicología. Madrid: Thomson.

Torres, M., Morocho, J., Santos, J., Escurra, L. M., Delgado, A. E., Quezada, R., Guevara, G. y Rivas, G. (2005). Relación entre el autoconcepto de las competencias, las metas académicas y el rendimiento en alumnos universitarios de la ciudad de Lima. Revista De Investigación En Psicología, 8(1) 87-106.

Villa, S.A. y Auzmendi, E.E. (1992). Medición del autoconcepto en la edad infantil. Bilbao: Ediciones Mensajero. 